\title{
MIDAS
}

Museus e estudos interdisciplinares

$6 \mid 2016$

Dossier temático: "Museus, discurso e poder"

\section{Memórias de exposições: o projeto RaisExpo}

Remembering exhibitions: the RaisExpo project

\section{Joana Baião}

\section{OpenEdition}

Journals

Edição electrónica

URL: http://journals.openedition.org/midas/969

DOI: 10.4000/midas.969

ISSN: 2182-9543

\section{Editora:}

Alice Semedo, Paulo Simões Rodrigues, Pedro Casaleiro, Raquel Henriques da Silva, Ana Carvalho

\section{Refêrencia eletrónica}

Joana Baião, « Memórias de exposições: o projeto RaisExpo », MIDAS [Online], 6 | 2016, posto online no dia 24 março 2016, consultado no dia 23 abril 2019. URL : http://journals.openedition.org/ midas/969; DOI : 10.4000/midas.969

Este documento foi criado de forma automática no dia 23 Abril 2019

\section{(c) (i) (2) (2)}

Midas is licensed under a Creative Commons Attribution-NonCommercial-ShareAlike 3.0 International License 


\title{
Memórias de exposições: o projeto RaisExpo
}

\author{
Remembering exhibitions: the RaisExpo project
}

Joana Baião

\section{História das exposições: uma nova disciplina?}

1 As exposições tornaram-se, desde a década de 90 do século passado, verdadeiros casos de estudo para museólogos e historiadores da arte. 0 crescente interesse pela sua história tem sido transversal ao universo académico e museológico anglo-saxónico e francófono. Esta abordagem tem dado azo a interessantes discussões historiográficas e metodológicas, sediadas na questão tão bem formulada, em 2010, pela curadora e crítica de arte contemporânea Judith Souriau: «dans quelle mesure l'histoire des expositions apparaitt comme un apport pertinent dans l'histoire de l'art, et surtout, comment elle se positionne par rapport à elle?» (Souriau 2010, s/p).

2 As respostas a esta questão não têm sido, de facto, consensuais. Por um lado, há uma corrente que entende a história das exposições de arte como disciplina complementar ou contígua da história da arte, uma «méta-histoire de l'art» (Souriau 2010, s/p). Por outro lado, há autores que defendem a autonomia disciplinar da história das exposições, verificando que esta é incompatível, epistemológica e metodologicamente, com a história da arte, devido às suas naturais diferenças: a história da arte está fundada na autonomia e perpetuidade do objeto artístico; a história das exposições tem um objeto de análise plural, efémero, cujo estudo tem de considerar a sua historicidade e especificidade espaço-temporal:

L'histoire des expositions n'est pas compatible avec l'histoire de l'art. Elle n'a pas le même champ, ni le même objet que l'histoire de l'art, et elle dérange les historiens d'art. (Glicenstein citado por Souriau 2010, s/p)

L'histoire de l'exposition n'est évidemment pas la même chose que l'histoire de l'art, ni que l'histoire des représentations, l'histoire des techniques, ou l'histoire des idées, mais emprunte nécessairement des éléments à toutes ces catégories (et à autres). En fait, l'histoire de l'exposition est un domaine à la fois incohérent par 
nature (car fortement hétérogène) et en permanence en chantier (...). Par certains aspects, une exposition est plus facile à définir dans l'espace et dans le temps qu'une œuvre d'art, alors que par d'autres, c'est un objet absolument insaisissable. (Glicenstein 2009, 241)

Discussões à parte - bem sintetizadas num artigo publicado por Parcollet e Szacka (2014) -, não deixa de ser consensual o reconhecimento da importância das exposições como objeto de estudo, na medida em que constituem o primeiro contexto em que as obras de arte são pela primeira vez apresentadas ao público. As exposições determinam o modo como a obra de arte é mostrada e recebida, influindo o seu impacto no panorama artístico geral, na crítica da arte e no mercado. Simultaneamente, pode ser reconhecida em determinadas exposições uma dimensão artística própria - «exhibits that exhibit themselves more than the content of their display» (La Rosa 2014, 1) -, definida quer pela intervenção mais explícita de arquitetos, museógrafos, designers ou artistas, quer pela qualidade e atualidade criativa, e, por vezes, vanguardista das montagens propostas.

4 Por fim, refira-se a importância das exposições como eventos passíveis de instrumentalização oficial ou propagandística, com potencial impacto na construção e fixação de narrativas historiográficas, na definição de medidas de proteção patrimonial e de apoio às artes, na definição de estratégias políticas ou diplomáticas, em vários níveis.

\section{Revisitar exposições: memória, documentação e catalogação}

5 Depois do interesse despertado no campo da história da arte pelas exposições que se destacaram por terem revelado artistas e movimentos marcantes - em suma, as «exposições que fizeram a História da Arte», de acordo com a expressão utilizada por Altshuler $(2009 ; 2013)$ na sua monumental obra, em dois volumes, Exhibitions that Made Art History -, tem-se assistido a uma ampliação do fenómeno de interesse por exposições passadas e pela evocação da sua memória. Esse fenómeno assume diversas materializações: remontagem de exposições (integradas no conceito proposto por La Rosa (2014) de «re-enactment»); apresentação de "exposições de exposições" (denominadas por Reesa Greenberg (2009) como «remembering exhibitions», «term for exhibitions that remember past exhibitions»); inventariação e catalogação sistemática de exposições passadas, a partir da atividade de determinada instituição ou personalidade - de que são exemplos maiores o projeto em curso Catalogue Raisonné des Expositions du Centre Pompidou ${ }^{1}$ e a publicação Harald Szeeman with by Through Because Towards Despite: Catalogue of all Exhibitions, 1957-2005 (2007), dedicada às exposições comissariadas pelo reconhecido curador de arte contemporânea, Harald Szeeman (1933-2005).

Uma vez que as exposições são eventos efémeros, cuja memória se desmultiplica por diversos registos, e sendo a publicação de catálogos daí resultante um testemunho apenas parcial da sua realização, coloca-se a questão de como preservar e, sobretudo, tornar acessível a sua história, os seus conteúdos, o seu impacto artístico e cultural e, finalmente, o seu valor patrimonial.

7 Tem sido no contexto académico, em articulação com os museus de arte, que se tem avançado verdadeiramente na valorização da exposição enquanto testemunho e «objeto cultural em si mesmo» (Parcollet e Szacka 2012, 107). Neste momento, o debate em torno das metodologias para a documentação e para a preservação da memória dos eventos 
expositivos avançou para uma nova fase, que põe em prática os procedimentos e as abordagens debatidos anteriormente.

Os projetos que se têm dedicado ao estudo sistemático de exposições optaram por abordálas enquanto objetos, aproximando-as de criações artísticas como as instalações ou performances, também de natureza efémera e condicionadas pelos locais onde são realizadas. Como consequência, cada exposição-objeto é alvo de um registo de inventário sistemático que, no seu conjunto e de acordo com objetivos pré-estabelecidos, pode resultar numa catalogação completa dos eventos expositivos (catálogo raisonné).

Os catálogos raisonné de exposições têm como ponto de partida o levantamento e análise de material de arquivo relacionado com cada um dos eventos estudados. De facto, uma das consequências do recente processo de valorização histórica das exposições prende-se com a igual valorização dos registos documentais a estas associados e, consequentemente, de toda a documentação gerada pela atividade dos museus. O material documental relacionado com as exposições é essencial para traçar o seu processo de conceção e organização, determinar o seu verdadeiro impacto e tornar esses eventos visualmente acessíveis no presente através da recolha de registos fotográficos e fílmicos. Neste campo refiram-se, por exemplo: a programação do Van Abbemuseum (Eindhoven, Holanda), que tem desenvolvido exposições centradas em material de arquivo, recriando em contexto expositivo modelos museográficos do passado e que, através do projeto Living Archive, se apresentaram exposições em que obras da coleção eram relacionadas com a documentação produzida no contexto da sua incorporação e gestão ${ }^{2}$; ou a exposição $L e$ Surréalisme et l'objet que teve lugar no Centro Cultural Pompidou entre outubro de $2013 \mathrm{e}$ março de 2014, cuja montagem se sustentava no diálogo entre a projeção audiovisual de material de arquivo e os objetos expostos, criando uma grelha de imagens poderosa e complexa entre o passado e o presente. ${ }^{3}$

\section{A Fundação Calouste Gulbenkian e o impacto das suas exposições no panorama artístico português}

10 A Fundação Calouste Gulbenkian (FCG) foi criada em 1956, por disposição testamentária de Calouste Sarkis Gulbenkian (1869-1955). Os seus estatutos foram aprovados pelo Estado português a 18 de julho de 1956, determinando que uma das suas áreas de atuação fosse a das artes plásticas. Esta finalidade artística coincidia não só com a biografia do Fundador, como também com um dos fundamentos da própria criação da FCG, uma vez que entre os seus objetivos estavam a conservação e a exibição, num único espaço, das obras que Gulbenkian colecionara ao longo da sua vida (Oliveira 2013a, 53).

11 Visando cumprir a sua missão artística, foi criado logo em 1956 o Serviço de Museu e Belas-Artes, cuja atividade incidia na planificação do futuro museu da Fundação e na divulgação da arte portuguesa e internacional. Dado o amplo leque das suas atribuições, em 1961 este serviço foi extinto, dando origem ao Serviço de Belas-Artes (que se manteve ativo até 2010) e ao Serviço do Museu, principais produtores das atividades de promoção artística da FCG, entre as quais as exposições. A FCG mantém em arquivo a documentação gerada por estes e outros organismos internos ${ }^{4}$, nomeadamente os processos originais das exposições que promoveu, subsidiou ou organizou.

12 Em 2007, a exposição 50 Anos de Arte Portuguesa ${ }^{5}$ tornou evidente a importância deste material documental para a história da arte e para a cultura portuguesa da segunda 
metade do século XX. Nesta mostra, para além de serem apresentados documentos do Arquivo do Serviço de Belas-Artes relacionados com as bolsas atribuídas aos artistas portugueses ${ }^{6}$ (relatórios de atividades que, em muitos casos, assumiram uma dimensão artística), foram evocados documentalmente alguns eventos expositivos organizados pela FCG que tiveram impacto no panorama artístico português, como as três edições da Exposição de Artes Plásticas (1957, 1961 e 1986) ou o ciclo 7 Artistas ao 10. Mês, iniciado em 1996. Para a preparação desta exposição, as comissárias analisaram, pela primeira vez, algum do material documental do arquivo do Serviço de Belas-Artes, numa abordagem historiográfica da documentação que, para além de inédita, suscitou o início do seu tratamento arquivístico, através do projeto Arquivo da Fundação Calouste Gulbenkian que, a partir de 2003, passara a gerir a documentação proveniente dos vários serviços da FCG. Foi, portanto, no contexto de uma exposição que se tornou clara a relevância da atividade expositiva da FCG no panorama artístico português e a necessidade de estudar sistematicamente o seu acervo documental.

Recentemente, as exposições da FCG foram alvo de trabalhos de investigação académicos relevantes: uma tese de doutoramento concluída (Oliveira 2013a) e outra em curso $^{7}$ e a criação de uma visita virtual à Exposição de Artes Plásticas de $1957^{8}$, concebida no âmbito do projeto Fontes para a História dos Museus de Arte em Portugal ${ }^{9}$. Em 2007, por ocasião da celebração dos 50 anos da FCG, Ribeiro (2007) destacou também as iniciativas expositivas desta instituição, ao analisar mais amplamente a atividade da FCG no campo artístico.

o prestígio e o impacto das exposições da FCG justificam, por si só, e enquanto eventos singulares, a sua análise. No entanto, são muito mais do que fenómenos pontuais, podendo ser encaradas como acontecimentos históricos, verdadeiros testemunhos da arte e da cultura portuguesa, marcantes para a promoção e desenvolvimento das práticas e políticas artísticas e culturais.

$\mathrm{Na}$ área das artes plásticas, as exposições da FCG constituíram a vertente de maior impacto da sua atividade junto do público. De facto, expressavam as políticas de promoção dos jovens artistas e de salvaguarda do património empreendidas pela fundação, e asseguravam a divulgação da arte internacional de diversas geografias e cronologias no nosso país.

Uma primeira abordagem histórica ao universo expositivo da FCG permite-nos verificar que a sua imagem e identidade institucional construiu-se de modo muito expressivo através das exposições, pelo que sempre foram significativos os recursos disponibilizados para a organização destes eventos. As exposições organizadas pelo Serviço de Museu, pelo Serviço de Belas-Artes e pelo Centro de Arte Moderna (CAM), nomeadamente nos anos de implantação destes organismos, destacavam-se pelo cuidado, qualidade e modernidade das soluções museográficas, bem como pelos seus programas curatoriais, tudo isto marcado pela intervenção de personalidades portuguesas de destaque na área da arquitetura, das artes, do design e da história da arte. De facto, desde o início a intenção era «fazer novo e melhor» (Perdigão 1957 citado por Oliveira 2013a, 23), por comparação com o que se fizera até então, no panorama expositivo português.

Por terem constituído - e constituírem ainda hoje - marcos da cultura e da arte portuguesa, o estudo sistemático das exposições da FCG permite acompanhar as várias tendências da produção artística da segunda metade do século XX, rever a historiografia da arte portuguesa, analisar a receção das tendências artísticas internacionais, e recuperar os discursos relacionados com a preservação patrimonial e promoção artística. Para além disso, sendo a organização de exposições acompanhada de iniciativas 
educativas, o estudo das exposições da FCG permite também traçar o desenvolvimento dos programas educativos associados à arte.

O reconhecimento da importância das exposições produzidas pela FCG desde a sua instituição, bem como a verificação da riqueza documental do seu arquivo, esteve na génese do projeto atualmente em curso: a criação de um catálogo raisonné das exposições de arte da FCG.

\section{O projeto RaisExpo - Catálogo Raisonné Online das Exposições de Arte da Fundação Calouste Gulbenkian}

Pela novidade e pelo contributo que as exposições da FCG trouxeram ao desenvolvimento da museografia, da arquitetura e do design de exposições em Portugal, estas podem ser encaradas - o distanciamento historiográfico permite esta abordagem -, como objetos artísticos em si mesmo, suscetíveis de nova análise e interpretação.

Tendo como exemplo o modelo estabelecido pelo projeto do Centro Georges Pompidou, acima mencionado, o projeto RaisExpo - Catálogo Raisonné Online das Exposições de Arte da Fundação Calouste Gulbenkian (daqui em diante RaisExpo) visa, em primeiro lugar, a inventariação, o estudo e a divulgação da memória expositiva da FCG no campo artístico. Simultaneamente, pretende explicitar a múltipla valência destes eventos expositivos, procurando ainda participar no debate que está a decorrer a nível internacional sobre a valorização destes eventos na análise crítica da história da arte, contribuindo para a definição de metodologias de valorização e patrimonialização das exposições.

O projeto RaisExpo arrancou em outubro de 2013, por iniciativa da presidência da FCG. Coordenado por Helena de Freitas e por Raquel Henriques da Silva, é um projeto transversal à colaboração das diferentes unidades orgânicas desta Instituição, que lhe estão tematicamente associados. Por esta razão, estabeleceram-se desde logo estreitas relações com o Arquivo e a Biblioteca de Arte, bem como com o Museu Calouste Gulbenkian e com o CAM. Além disso, foi celebrada uma parceria estratégica com o Instituto de História da Arte da Faculdade de Ciências Sociais e Humanas da Universidade Nova de Lisboa. Resultou, deste modo, uma relação entre a FCG, protagonista de uma ação relevante no panorama artístico português e detentora de fontes essenciais para documentar a arte portuguesa da segunda metade do século xx, e uma unidade de investigação que promove a interpretação desse material documental, valorizando-o enquanto fonte para a produção de novo conhecimento e de novos modos de perspetivar a história da arte portuguesa.

Sendo o ponto de partida do projeto o estudo da documentação produzida pelos vários serviços que, dentro da FCG, organizaram exposições, tornou-se desde logo importante clarificar a estrutura orgânica desta instituição. Verificou-se que a realização de exposições tem sido transversal a vários serviços da fundação: Serviço de Belas-Artes, Serviço do Museu, Serviço de Exposições e Museografia, CAM. Outros serviços não relacionados com as artes plásticas, como o Serviço de Música ou a área das Ciências da FCG também desenvolveram projetos expositivos. Tendo presente esta informação, e verificando-se a multiplicidade e diversidade temática das exposições realizadas pela FCG, o projeto RaisExpo optou, pelo menos numa primeira fase de trabalho, por sistematizar informação unicamente relativa às exposições de arte, produzidas pelo Serviço de Belas- 
Artes, Serviço do Museu, Serviço de Exposições e Museografia, CAM, e também pelas delegações francesa e inglesa da FCG.

Definido este ponto, e tendo como base o entendimento de cada evento expositivo como objeto inventariável, partiu-se para a construção da «ficha de exposição», a partir da questão: que dados deverão ser sistematicamente recolhidos, inventariados e, depois, analisados? A definição da "ficha de exposição»" foi fruto de um longo processo de reflexão por parte da equipa do projeto, e teve em consideração a extensa cronologia em análise (1957 até à atualidade), a complexidade da estrutura e da atividade expositiva da FCG e as fontes disponíveis em arquivo e na biblioteca.

24 A inventariação das exposições da FCG está a ser integrada numa plataforma informática, o In Arte - base de dados que já servia o inventário da coleção do Museu Calouste Gulbenkian e do CAM, e que continha já uma tarefa dedicada às exposições. Adaptaram-se os campos já existentes e adicionaram-se novos campos, de acordo com as necessidades do projeto.

O acesso público à base de dados do projeto RaisExpo será feito online, através de um website. Para além de permitir a consulta da ficha de inventário de cada exposição, com uma pequena descrição de cada evento, este website permitirá o acesso a dados gerais, de natureza mais estatística, sobre o conjunto das atividades expositivas organizadas pela FCG: locais (em sistema de geo-referenciação), instituições parceiras, quadro cronológico, temas, artistas participantes, obras expostas, etc. Além disso, haverá uma componente visual bastante forte, sustentada quer pelo vasto acervo fotográfico da Fundação, quer pelo relevante material gráfico (cartazes, capas de catálogos, convites) e audiovisual produzido ao longo dos quase 60 anos de atividade expositiva. Após a implementação do website, prevista para 2016, os dados serão disponibilizados de modo progressivo, acompanhando o avanço da investigação.

A conclusão do projeto está prevista para 2019, ano comemorativo do 50. aniversário da inauguração da sede e museu da FCG, em que serão organizados eventos que darão a conhecer os resultados deste trabalho.

Como nota conclusiva refira-se que, à data da redação deste texto, dois anos volvidos sobre o início do projeto RaisExpo, pode ser feito um primeiro balanço sobre a(s) atividade(s) expositiva(s) da FCG, principalmente nos primeiros anos da sua existência. Reforçando as conclusões de Oliveira (2013a; 2013b), que defende que as exposições de arte foram um instrumento privilegiado pela Fundação para construir e consolidar a sua imagem no contexto nacional e internacional, verificamos que essa instrumentalização se relacionou diretamente com a sua missão filantrópica (apoio às artes e à investigação) e com a sua estratégia diplomática interna e externa (relações com instituições portuguesas e estrangeiras). Isso refletiu-se desde logo numa dupla movimentação - de dentro para fora e de fora para dentro - que, no campo das exposições, teve expressão na organização de importantes mostras de arte portuguesa no estrangeiro e, simultaneamente, na apresentação em Portugal de alguns dos mais significativos movimentos artísticos internacionais, numa clara estratégia de proporcionar ao público português um inédito contacto com os períodos mais importantes da história da arte internacional. A par destes movimentos, deve ser ainda destacado o papel da Fundação no apoio aos artistas portugueses, não só por via dos subsídios de estudo, mas também por via da aquisição das suas obras, muitas vezes em contexto de exposições individuais ou coletivas organizadas ou subsidiadas pela própria FCG, em Portugal ou no estrangeiro ${ }^{11}$. 
estes temas associados ao estudo das exposições da FCG e ao intenso trabalho que tem vindo a ser desenvolvido pelo projeto RaisExpo, revelam a importância de projetos de investigação desta natureza, que privilegiam não só a identificação e sistematização da informação de fontes primárias e secundárias, mas que visam também contribuir para a sua interpretação e análise, concorrendo para novas reflexões históricas e críticas acerca da arte e da cultura do século XX.

Por fim, refira-se que o projeto RaisExpo está aberto à comunidade científica, tendo vindo a acolher estágios curriculares de alunos da licenciatura em História da Arte, bem como projetos individuais de mestrado em História da Arte e em Museologia ${ }^{12}$. É objetivo deste projeto alargar e fortalecer os laços com as instituições académicas, pelo que se continuará a receber projetos de alunos e investigadores que queiram desenvolver estudos sobre temas que se cruzem, direta ou indiretamente, com o vasto universo das exposições de arte da FCG.

\section{BIBLIOGRAFIA}

Altshuler, Bruce. 2009. Exhibitions that Made Art History. Vol. 1: 1863-1959 - Salon to Biennial. London: Phaidon Press.

Altshuler, Bruce. 2013. Exhibitions that Made Art History. Vol. 2: 1962-2002 - Biennials and Beyond. London: Phaidon Press.

Glicenstein, Jérôme. 2009. L'Art: Une Histoire d'Expositions. Paris: Presses Universitaires de France. Greenberg, Reesa. 2009. “'Remembering Exhibitions': From Point to Line to Web.” Tate Papers Issue 12 (Autumn).

La Rosa, Miriam. 2014. “'Re-enacting Exibitions': A Case-study. Stephen Willats Between Raven Row and the Whitechapel Gallery." MACC Curating the Contemporary 2013-14: Theory in Practice 1-14.

Matias, Carolina Gouveia. 2015. “A III Exposição de Artes Plásticas da Fundação Calouste Gulbenkian. Julho-Setembro 1986." Relatório de estágio no âmbito do mestrado em Museologia, Faculdade de Ciências Sociais e Humanas da Universidade Nova de Lisboa.

Oliveira, Leonor de. 2013a. "Fundação Calouste Gulbenkian: Estratégias de Apoio e Internacionalização da Arte Portuguesa 1957-1969". Tese de doutoramento em História da Arte, especialização em Museologia e Património Artístico, Faculdade de Ciências Sociais e Humanas da Universidade Nova de Lisboa.

Oliveira, Leonor de. 2013b. "Politics, Diplomatic Relations and Institutional Promotion through Modern Art - the British Art of the Twentieth Century Exhibition in Portugal, 1962." RIHA Journal 0072 (19 July).

Parcollet, Rémi, e Léa-Catherine Szacka. 2012. "Histoire des Expositions du Centre Pompidou: Réflexions sur la Constitution d'un Catalogue Raisonné." Marges. Revue d'Art Contemporain 15: 107-127. 
Parcollet, Rémi, e Léa-Catherine Szacka. 2014. "Écrire l'Histoire des Expositions: Réflexions sur la Constitution d'un Catalogue Raisonné d'Expositions." Culture \& Musées 22 (janvier) (Documenter les Collections, Cataloguer l'Exposition, direção de Gérard Régimeau): 137-162.

Ribeiro, António Pinto. 2007. “Arte. Um Ministério das Artes: Das Belas-artes, das Exposições, dos Subsídios, do Teatro, do Cinema e das Bolsas." In Fundação Calouste Gulbenkian: Cinquenta Anos, 1956-2006, 237-408. Lisboa: Fundação Calouste Gulbenkian.

Silva, Raquel Henriques da, Joana Baião, e Leonor de Oliveira, eds. 2013. Projetha. Projects of the Institute of History of Art. Sources for the History of Art Museums in Portugal - Final Report. Lisboa: Instituto de História da Arte, Faculdade de Ciências Sociais e Humanas da Universidade Nova de Lisboa.

Souriau, Judith. 2010. “L'Histoire des Expositions: Une Nouvelle Histoire de l'Art?" Association Thes-Arts. Consultado em dezembro 15, 2013. http://www.thes-arts.com/index.php? option=com_content\&view=article\&id=125\&Itemid=59.

Szeeman, Harald. 2007. "Harald Szeeman with, by, Through, Because, Towards, Despite: Catalogue of all Exhibitions, 1957-2005, editado por Tobia Bezzola e Roman Kurzmeyer. Vienna: Springer.

\section{NOTAS}

1. http://goo.gl/psemmr (consultado em junho 26, 2015).

2. Living Archive: http://goo.gl/eqcOHB (consultado em junho 26, 2015). A programação expositiva - atual e passada - deste museu pode ser consultada em: http://vanabbemuseum.nl/ en/ (em inglês).

3. Informações sobre a exposição em: https://www.centrepompidou.fr/cpv/resource/ccAyeG/ rBAEd6g (consultado em junho 26, 2015).

4. Para além do Serviço de Belas-Artes e do Serviço do Museu, tiveram particular impacto na produção ou montagem de exposições da FCG - gerando, por isso, documentação relativa a estes eventos - o Serviço de Projetos e Obras (criado em 1956) e o Serviço de Exposições e Museografia (criado em 1969), ambos integrados mais tarde nos Serviços Centrais da fundação, e o Centro de Arte Moderna (inaugurado em 1983).

5. Exposição realizada na galeria de exposições temporárias do Museu Calouste Gulbenkian, comissariada por Raquel Henriques da Silva, Ana Filipa Candeias e Ana Ruivo.

6. O SBA foi responsável, para além da organização de exposições, pela gestão do programa de bolsas a artistas, historiadores e críticos de arte portugueses, entre 1960 e 2010.

7. A tese de doutoramento em curso é de André da Silveira Rodrigues, intitulada: Um Encontro Possível da Arte na Europa: A Exposição-Diálogo de 1985.

8. http://expo1957.fcsh.unl.pt/FCTProject/faces/Index.xhtml (consultado em junho 26, 2015). A criação da visita virtual à exposição da FCG foi fruto da parceria com o Centro de Investigação em Informática e Tecnologias da Informação - CITI, da Faculdade de Ciências e Tecnologia da Universidade Nova de Lisboa.

9. Projeto financiado pela Fundação para a Ciência e a Tecnologia e coordenado por Raquel Henriques da Silva (PTDC/EAT-MUS/101463/2008); teve por objetivo o levantamento, estudo e disponibilização de fontes documentais relacionadas com a história dos museus de arte em Portugal (v. Silva, Baião e Oliveira 2013).

10. A ficha de exposições da FCG integra os seguintes campos: título; local e data de realização; data de produção; participantes; ficha técnica (serviço responsável; comissários; designers; instituições parceiras; etc.); tipo de exposição; temas; orçamento; n.․ de visitantes; eventos 
associados; documentação e bibliografia relacionados (documentos; material gráfico; material fotográfico; material audiovisual; periódicos; monografias; outros). Para além destes campos, serão relacionadas às respetivas exposições as obras da coleção FCG expostas e/ou incorporadas no seu contexto. Foram definidos campos de acesso público e reservado, que terá repercussões quando a base de dados for disponibilizada ao público.

11. Estes temas foram alvo de uma primeira análise na comunicação «Expor lá Fora: a Fundação Calouste Gulbenkian e a Divulgação Internacional da Arte Portuguesa na Década de 1960», da autoria de Filipa Coimbra e Joana Baião. Esta comunicação foi apresentada no âmbito do Encontro MuSt (Museum Studies), dedicado ao tema Nacionalidade, Identidade, Mobilidade: Geopolítica e Exposições de Arte e que teve lugar na Faculdade de Belas-Artes da Universidade do Porto a 27 de novembro de 2015.

12. Refira-se a conclusão, em janeiro 2016, do primeiro estágio de mestrado em Museologia realizado no âmbito do projeto, que teve como resultado o relatório A III Exposição de Artes Plásticas da Fundação Calouste Gulbenkian: Julho-Setembro 1986, de Carolina Gouveia Matias. Este trabalho, inscrito na Faculdade de Ciências Sociais e Humanas da Universidade Nova de Lisboa, foi orientado por Leonor de Oliveira e co-orientado por Helena de Freitas.

\section{RESUMOS}

A história das exposições tornou-se num dos temas atualmente mais discutidos e valorizados nos campos da história da arte e da museologia. Esta nova disciplina está a contribuir para uma revisão crítica da historiografia da arte, das práticas museológicas e das políticas de promoção artística e valorização patrimonial. o projeto Catálogo Raisonné Online das Exposições de Arte da Fundação Calouste Gulbenkian insere-se no amplo debate internacional em torno do tema das exposições, participando numa tendência recente de valorização patrimonial destes eventos e de salvaguarda da sua memória. Neste artigo damos a conhecer os objetivos, a estrutura e a metodologia de trabalho deste projeto, tendo em conta os desafios que se colocam à valorização e divulgação deste património.

The history of exhibitions has recently become one of the most discussed and relevant themes in the fields of Art History and Museology. This new field is contributing to a critical review of art historiography and museological practices as well as artistic promotion and heritage preservation policies. The Online Catalogue Raisonné of the Calouste Gulbenkian Foundation's Art Exhibitions integrates a wider international debate related to exhibition studies, and is participating in the recent process of patrimonialization and memory preservation of these events. This article introduces the goals, structure and methodology of the project, pointing out current challenges facing the promotion of such heritage.

\section{ÍNDICE}

Keywords: exhibition, Calouste Gulbenkian Foundation, inventory, memory

Palavras-chave: exposição, Fundação Calouste Gulbenkian, inventário, documentação, memória 


\section{AUTOR}

\section{JOANA BAIÃO}

Membro integrado do Instituto de História da Arte da Faculdade de Ciências Sociais e Humanas da Universidade Nova de Lisboa. Licenciada em Escultura pela Faculdade de Belas-Artes da Universidade de Lisboa (2005), mestre em Museologia pela Universidade Nova (2009) e doutora em História da Arte - especialização em Museologia e Património Artístico na mesma Universidade (2014). Tem colaborado com várias instituições culturais portuguesas (Museu Nacional de Arte Contemporânea - Museu do Chiado; Museu Coleção Berardo; Fundação Arpad Szenes - Vieira da Silva). Atualmente, é assistente de coordenação do projeto RaisExpo - Catálogo Raisonné Online das Exposições de Arte da Fundação Calouste Gulbenkian. jbaiao@gulbenkian.com 